\title{
Design of neuro-simulation system in situational management of control and quality assessment for complex production assembly system
}

\author{
A. L. Zolkin ${ }^{1}$, E. A. Lavrov $^{2}$, I. N. Zaitseva ${ }^{3}$, A. S. Bityutskiy ${ }^{4}$ and V. A. Mironchuk ${ }^{5}$ \\ ${ }^{1}$ Computer and Information Sciences Department, Povolzhskiy State University of Telecommunications and \\ Informatics, Samara, 443010, Russia \\ ${ }^{2}$ Computer science department, Sumy State University, Sumy, 40007, Ukraine \\ ${ }^{3}$ Department of Physics, Radio Engineering and Electronics, Bunin Yelets State University, Lipetsk oblast, Elets, \\ 399770, Russia \\ 4"Invent Technology" LLP, Almaty A10E5P4, Kazakhstan \\ ${ }^{5}$ Department of Economic Cybernetics, Federal State Budgetary Educational Institution of Higher Education \\ “Kuban State Agrarian University named after I.T. Trubilin”, Krasnodar, 350044, Russia
}

\begin{abstract}
The specific challenge for application of developed system of situational input in control process is generalized by authors, flowgraph model is demonstrated, synthesis methodology is generalized and assessment of effectiveness based on group of parameters of regression nature to implemented neuro-fuzzy regulation system is described. Decomposition and structural analysis of specificity of application of implemented model, its characteristic function and balance-model (before implementation) are described. The steps of development of this complex technology are reviewed.
\end{abstract}

\section{Keywords}

Neuro-simulation, fuzzy control, situational control, non-direct input, additive technologies, automation of technological production

\section{Introduction}

The process of differentiation of control for complex technical systems - the searching of regulation method for control system in the context of application in automated assembly line for system of elevator type with complicated multi-position tools of control is used in this study as studying object and formalization of object of challenge definition [1].

The organization and technical complex performing discrete number of operations on unlimited (analogue) space of input values or parameters accepting and registering by system of perception (input system) is meant under complex technical system.

Control of complex technical systems is one of the key challenge cybernetic and reviewed as specific goal of cybernetic cooperation. Complexity of goal is explained with object and structure variety of such complexes which are socially oriented by assignment of some systems $[2,3]$.

\footnotetext{
Proceedings of MIP Computing-V 2022: V International Scientific Workshop on Modeling, Information Processing and Computing, January 25, 2022, Krasnoyarsk, Russia

EMAIL: alzolkin@list.ru (A. L. Zolkin); prof_lavrov@mail.ru (E. A. Lavrov); irina-zai73@mail.ru (I. N. Zaitseva); bsalexey@mail.ru (A. S. Bityutskiy); va_mironchuk@mail.ru (V. A. Mironchuk)

ORCID: 0000-0001-5806-9906 (A. L. Zolkin); 0000-0001-9117-5727 (E. A. Lavrov); 0000-0003-3415-2099 (I. N. Zaitseva); 0000-0002-

2754-1531 (A. S. Bityutskiy) 0000-0001-9160-4704 (V. A. Mironchuk)

(C) (i) (C) 2022 Copyright for this paper by its authors.

Bs - Usermitted under Creative Commons License Attribution 4.0 International (CC BY 4.0).

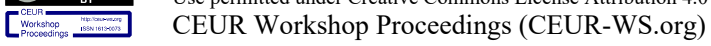




\section{Problem statement}

In reviewed study we have to characterize in form of challenge statement the process of functionality of technical complex of control of elevator stations control on the warehouse of big distributor. We should assign the complexity of reviewed system from the position of structural analysis: it is assumed the logistic of item from point "A" to point "B" by cross-movements in space taking into account the switching on different types of surface and production breaks (such challenge is solved in general by traditional systems of automotive control systems) as well as role-based significance of combining the control way with moving the elevator line (line system) and with assurance of unity of verification of assembly process is emphasized and stated [4]. Even though the reviewed models [5,6,7] of application of situational control allow to assign the conditions of company's operation and evaluate the potential risks but they do not define the technical organization on already presented control systems. However, the reviewed technical system is significantly complicated from the position of working principles.

\section{Research questions}

Assembly system of company has a form of iteration algorithm of technology on additive (composition) type, i.e. consists of some independent processes connecting with each other through delegation tool on the stage of assembly. The main time period company is involved in logging of wood incoming from neighboring sites where the system of processing the raw material - wood and different materials (epoxide, wood dust, round wood) - is performed.

Before going to the optimization challenge description it is required to reduce the informational entropy about studying object - assembly line [8].

Before reviewing the system of assembly lines (having the form chain of contours of production associated on functional presentation as well as on structure one) we should to explain the common characterization function on the example of specific line.

For this purpose, we should refer to formalized presentation of graph-transferring line of company on Figure 1.

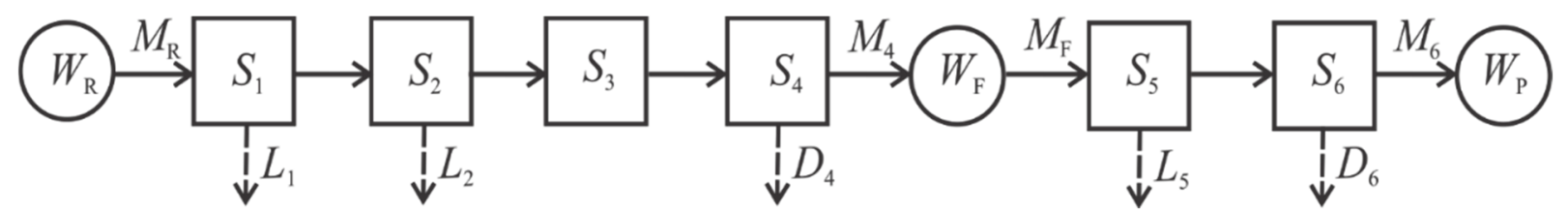

Figure 1: Flowgraph model of production of specific type of product (parquet block from ash in polygonal format)

Perform the decomposition of flowgraph model and some summarizing of group of activating functions of production assembly line of elevator type (see Table 1).

Table 1

Decomposition of activating functions of production

\begin{tabular}{ccc}
\hline Iteration & $\begin{array}{c}\text { Operation descriptor } \\
\text { (Semantic description) }\end{array}$ & Unit of measurement \\
\hline WR & Raw material storekeeping & Cube metres \\
S1 & Raw material cut & Cube metres \\
S2 & Manufacturing & units \\
S3 & Semi-finished product formation & units \\
S4 & Sorting of parquet boards & units \\
WM & Storekeeping of parquet boards & units \\
& Assembly process of parquet product & units \\
S5 & Engineering conversion & units
\end{tabular}


As it was mentioned before, group of events combined in functional group is reviewed in this study; at the same time, for example, stage of "sorting and incapsulation of parquet" is not a compatible process performed on previous and next stages, i.e. the strong specific section of assembly line and procedure of switching are mentioned [9].

\section{Materials and methods}

Linguistic methods of initiation of numerical variables membership from theory of fuzzy control and simulation are a basis of developed control method. The approaches presented in modern applications in area of compositional hybrid simulation were used as materials associated in process of complex method synthesis [10].

Semantic components defined to rank conditions applicable to specific technological process or group of events affected on process of decision making directing on specific control action or functional decision based on situation graphs for acceptance of specific approximative action from linguistic variable used as identifying the trueness of result for finish action were used as data which are object of storage, analysis and processing.

Two approaches to neuro-simulation system organization are combined in developed control method:

- the physical nature of proceed event is taken into account in first approach, i.e. the value of engineering mechanics, section of physics described the ratio of object unreactiveness, as well as dynamics of material point in the context of reviewed complex technical system is identified [11];

- cybernetic approach indirected by systematic analysis and technical design specification is used in second approach.

Method of direct inputs from algorithmization was mentioned here directly with neuro-fuzzy regulator model.

Defined neuro-fuzzy system was based on idea of modified perceptron. The work on modificator was done within several stages in the frame of consistent work of group of authors. Situational model resolved into direct inputs described by fuzzy rules with activating function and calculation of regressions of compliance of estimated operation rules and standard values of outputs from assembly line [12].

\section{Results}

The subtraction circuit of activating values based on voltage level (instantaneous voltage) on windings power stations of contour (situational model becomes effective in case of combination specific levels of voltage in system as they specifically represent modulate characteristic of operation scenario of complex assembly line (based on group of working parameters - presenting volume and quality of work) is defined and demonstrated in Figure 2.

The values from balanced balance-model based on which the calculation was done before start of work (with the aim to identify the linear regression at the moment of functionality of current control system) were assigned as econometric data base used for study (metrics of effectiveness and correlation of system components assessment). 


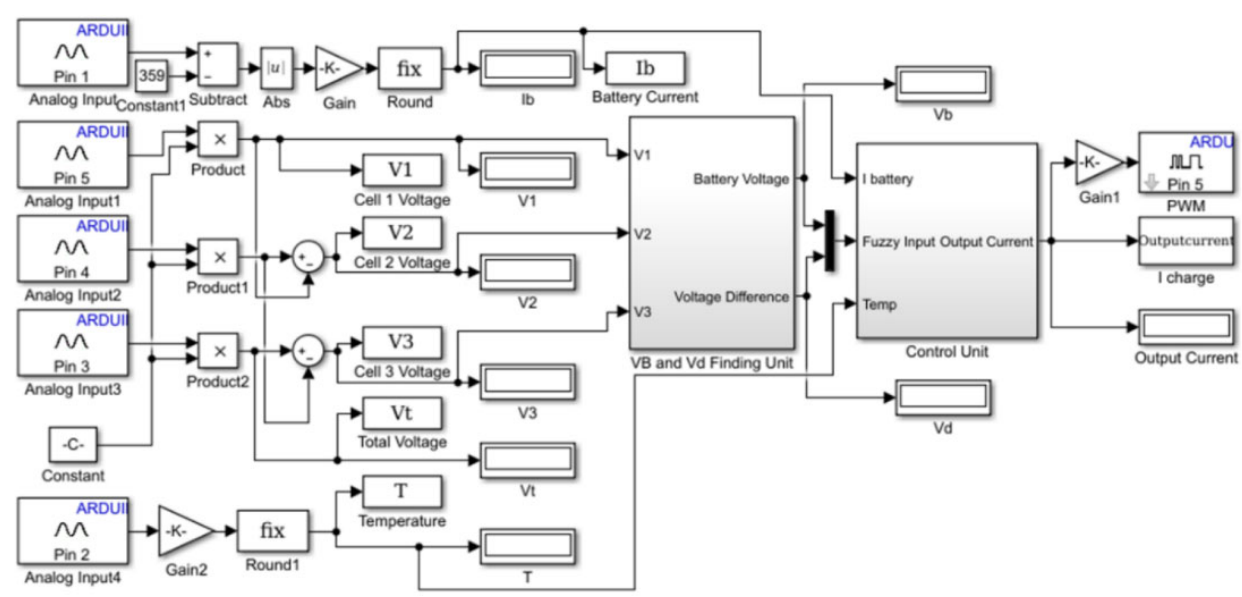

Figure 2: Neuro-fuzzy independent system of regulation for assembly line

The uniform variances on criteria of number of assembled products for efficient cycle of work (active equipment of assembly line operates with technological breaks each 30 minutes of continuous work when the situational algorithm based on heuristic approach, i.e. defining the need of switching of context of variability) are presented as values in basic Table 2 .

\section{Table 2}

Balance-model of production effectiveness of line in case of switching to other types of product's assembly with programming algorithm

\begin{tabular}{cccccc}
\hline \multirow{2}{*}{$\begin{array}{c}\text { Number of products, } \\
\text { units }\end{array}$} & 1 & 2 & 3 & 4 & 5 \\
\cline { 2 - 6 } & 10 & 8 & - & - & - \\
\hline 14 & - & 12 & 7 & - & - \\
15 & - & - & 28 & 6 & - \\
16 & - & - & - & 8 & 9 \\
17 & - & - & - & - & 12 \\
18 & & & & &
\end{tabular}

We should calculate the sample coefficient of correlation and determination on the basis of this data for direct control before implementation of situational simulation and find the sample equation of linear regression that describes the correlation dependence of $Y$ value (current scenario of control) from $X$ quantity. Task solution of characterization resolve into composition of correlation table, (ny, nx- value frequencies $\mathrm{x}$ and $\mathrm{y}$ correspondingly, $\mathrm{n}$ - sample volume) (Table 3).

\section{Table 3}

Correlation table (for direct scenario of work)

\begin{tabular}{ccccccc}
\hline $\mathrm{Y}$ & \multicolumn{7}{c}{$\mathrm{X}$} \\
\cline { 2 - 7 } & 1 & 2 & 3 & 4 & 5 & $\mathrm{n}_{\mathrm{v}}$ \\
\hline 14 & 10 & 8 & - & - & - & 18 \\
15 & - & 12 & 7 & - & - & 19 \\
16 & - & - & 28 & 6 & - & 34 \\
17 & - & - & - & 8 & 9 & 17 \\
18 & - & - & - & - & 12 & 12 \\
$\mathrm{n}_{\mathrm{x}}$ & 10 & 20 & 35 & 14 & 21 & $\mathrm{n}=100$ \\
\hline
\end{tabular}


Find $\bar{x}$ and $\bar{y}$ for direct mode of work (formulae 1,2) and corresponding characteristics (formulae $3,4,5,6,7,8,9)$, manually, to demonstrate later the finish results of calculations in case of using the apparatus of situational input in production process monitoring:

$$
\begin{gathered}
\bar{x}=\frac{1}{n} \sum_{i=1}^{5} x_{i} n_{x_{i}}=\frac{1}{100}(1 \cdot 10+2 \cdot 20+3 \cdot 35+4 \cdot 14+5 \cdot 21)=3.16 \\
\bar{y}=\frac{1}{n} \sum_{i=1}^{5} y_{i} n_{y_{i}}=\frac{1}{100}(14 \cdot 18+15 \cdot 19+16 \cdot 34+17 \cdot 17+18 \cdot 12)=15.86
\end{gathered}
$$

Calculate the sampling variance $D_{x}$, and then $\widetilde{\sigma}_{x}$ :

$$
\begin{gathered}
D_{x}=\frac{1}{n} \sum_{i=1}^{5}\left(x_{i}-\bar{x}\right)^{2} n_{x_{i}}=\frac{1}{100}\left((1-3.16)^{2} \cdot 10+(2-3.16)^{2} \cdot 20+(3-3.16)^{2} \cdot 35+\right. \\
\left.+(4-3.16)^{2} \cdot 14+(5-3.16)^{2} \cdot 21\right)=1.554 \\
\tilde{\sigma}_{x}=\sqrt{D_{x}}=\sqrt{1.554}=1.25
\end{gathered}
$$

Receive the $\tilde{\sigma}_{y}=1.24$. Find the sum:

$$
\begin{gathered}
\sum n_{x y} x y=(10 \cdot 1+8 \cdot 2) \cdot 14+(12 \cdot 2+7 \cdot 3) \cdot 15+(28 \cdot 3+6 \cdot 4) \cdot 16+ \\
+(8 \cdot 4+9 \cdot 5) \cdot 17+12 \cdot 5 \cdot 18=5156
\end{gathered}
$$

Find the unknown sample coefficient of correlation:

$$
r_{B}=\frac{\sum n_{x y} x y-n \bar{x} \bar{y}}{n \widetilde{\sigma}_{x} \widetilde{\sigma}_{y}}=\frac{5156-100 \cdot 3.16 \cdot 15.86}{100 \cdot 1.25 \cdot 1.24}=0.93,
$$

Therefore, $r_{B}=0.93$.

Coefficient of correlation shows that there is a strong connection between complex index of effectiveness [10] and number of switching.

Coefficient of determination $r^{2}=(0.94)^{2}=0.884$ shows that $88.4 \%$ of total effectiveness variation in work is caused by number of minimal switching, and (11.6\%) - by other factors which were not taken into account in this task. Minimization of $11 \%$ factors should be the finish results of this study.

b) substituted the founded values in equation

$$
\bar{y}_{x}-\bar{y}=r_{B} \frac{\tilde{\sigma}_{y}}{\widetilde{\sigma}_{x}}(x-\bar{x})
$$

Unknown equation of linear regression $\mathrm{Y}$ to $\mathrm{X}$ will be received:

$$
\bar{y}_{x}-15.86=0.93 \cdot \frac{1.24}{1.25}(x-3.16) \text {, }
$$

or finally $\bar{y}_{x}=0.923 x+12.94$.

So, equation of linear regression

$$
\overline{y_{x}}=a+b x=12.94+0.923 x
$$

\section{Findings}

Finally, the coefficient of regression $b=0.923$ shows that in case of increasing of number of switching of assembly line (x) on one determinant of working cycle (y) it is increased on 0.923 conditional units in average.

In case of implementation of situational model, the coefficient of regression takes the average value (24 versions of work simulation were processed) laying in range of 0.964-0.98 that, generally, presents the three sigma rule in normal distribution and shows the final objective of optimization. Therefore, 
realization of situational monitoring improves the quality and operation speed of complex assembly line on $9-19 \%$ under condition of using the different ways of assembling/ packaging of different products.

\section{Discussion}

During development of situational model, it is taken into account that management of parquet production is realized with the following mutually exclusive parameters composited in function (how the MUL-fuzzy output of information about control action is explained and that is presented in finish element of scheme in Figure 1).

The values themselves are determined into functional groups of parameters, where:

- $\quad$ MR- consumption of one type of wood material;

- L1 - losses of first class production during cut;

- L2 -losses of materials;

- $\quad$ D4 -waste of frieze;

- M4- income of frieze;

- MF- consumption of frieze;

- L5- total losses (rejects);

- D6- waste of product;

- M6- income of parquet.

Values of group L are the non-recoverable technological losses of raw material which are calculated based on the normative coefficients for each type of wood approved in company [12].

\section{Conclusion}

Taking into account the specific features of control practice in multi-stage production systems, the wide application for tasks of control with them of profitability analysis models based on assessment of level of correlation change from situational expenses can be confirmed [13].

Built model of development with next equal transformation to principle (method of correlated interaction) showed the functional balance and a context based analysis allowed to solve the task of situational monitoring at production that demonstrated the economic effectiveness of solution [14].

Demonstrated way of complex-system creation for management tool in conditions of indirect and periodic control allow to create and evaluate the new classes of information analysis systems for systems with tools - functional and regression nature in which the nature of compliance of expected functionality with actual compliance of work scenario which is not dissimilar with behavior of discontinuity function (discontinuous function) is stated and emphasized. The need of working out the common decisions on finding the discontinuity points - points of determination (direct switching) for specific technological processes in variety of dynamics of their demonstration is still remained.

\section{References}

[1] V. V. Borisov, D. Y. Avramenko, Fuzzy situational control of complex systems on the basis of their compositional glued simulation, Systems of control, connections and safet 3 (2021) 207-237.

[2] I. V. Kotenko, I. B. Parashchuk, Fuzzy control of information and security events: features of membership function building, Vestnik of Astrakhan State Technical University. Library: Management, Computer and Information Science 3 (2021) 7-15.

[3] N. O. Zagibin, S. V. Ulyanov, Program realization of fuzzy logic with linguistic variables, System analysis in science and education 1 (2021) 45-57.

[4] A. V. Mykhin, Optimum stabilization of rotor in electromagnetic suspension system with TakagiSugeno fuzzy models, Computer science challenges 2(51) (2021) 26-37. 
[5] V. V. Borisov, D. Y. Avramenko, Fuzzy situational control of complex systems on the basis of their compositional glued simulation, Systems of control, connections and safety 3 (2021) 207237.

[6] V. V. Oznamenec, Soft situational control, Slavyanskiy forum 2 (2018) 57-62.

[7] W. Chi et al., Design and experimental study of a VCM-based Stewart parallel mechanism used for active vibration isolation, Energies 8(8) (2015) 8001-8019.

[8] A. G. Burda, S. N. Kosnikov, V. I. Polusmak, S. A. Burda, Automation of dairy herd management and evaluation of its economic efficiency using an information system, IOP Conference Series: Earth and Environmental Science 624(1) (2021) 012144.

[9] F. Delfani, H. Samanipour, H. Beiki, A. Yumashev, E. Akhmetshin, A robust fuzzy optimisation for a multi-objective pharmaceutical supply chain network design problem considering reliability and delivery time, International Journal of Systems Science: Operations and Logistics 1-25 (2020). doi: 10.1080/23302674.2020.1862936.

[10] A. N. Riakhovskiǐ, S. I. Zheltov, V. A. Kniaz', A. V. Iumashev, A hardware and software complex for producing 3D models of the teeth Apparatno-programmnyi kompleks polucheniia 3D-modelei zubov, Stomatologiya 79(3) (2000) 41-45.

[11] A. M. Gubernatorov, M. S. Chistyakov, Convergence of digital technologies and industrial potential of manufacturing industries in the formation of the platform approach "Industry 4.0", Management of the economy: methods, models, technologies: materials of the XX International Scientific Conference. - Ufa: Ufa State Aviation Technical University 62-65 (2020).

[12] A. Vlasyuk, V. Zhukovskyy, N. Zhukovska, S. Shatnyi, Parallel Computing optimization of TwoDimensional Mathematical Modeling of Contaminant Migration in Catalytic Porous Media, 2020 10th International Conference on Advanced Computer Information Technologies, ACIT 2020 Proceedings, 2020, pp. 23-28, 9208878.

[13] D. I. Fakhertdinova, V. D. Munister, A. L. Zolkin, A. V. Knishov, M. Yu. Speranskiy, Application of discrete mathematics, tetralogic and architecture of superscalar systems in measurement metrology of automated control systems, IOP Conference Series. Krasnoyarsk Science and Technology City Hall. Krasnoyarsk, Russian Federation 22009 (2021). DOI: 10.1088/1742$6596 / 1889 / 2 / 022009$.

[14] V. S. Tormozov, A. L. Zolkin, K. A. Vasilenko, Optimization of neural network parameters based on a genetic algorithm for prediction of time series, 2020 International Multi-Conference on Industrial Engineering and Modern Technologies, FarEastCon 2020. 9271536 (2020). DOI: 10.1109/FarEastCon50210.2020.9271536. 